\title{
ON SMALL CHARACTERISTIC ALGEBRAIC TORI IN PAIRING-BASED CRYPTOGRAPHY
}

\author{
R. GRANGER, D. PAGE AND M. STAM
}

\begin{abstract}
The value of the Tate pairing on an elliptic curve over a finite field may be viewed as an element of an algebraic torus. Using this simple observation, we transfer techniques recently developed for torus-based cryptography to pairing-based cryptography, resulting in more efficient computations, and lower bandwidth requirements. To illustrate the efficacy of this approach, we apply the method to pairings on supersingular elliptic curves in characteristic three.
\end{abstract}

\section{Introduction}

The use of pairings in cryptography is now a well-studied area, with resulting applications to identity-based encryption, key-agreement and signature schemes [5, 44], tripartite DiffieHellman key-agreement [29], and short signatures [6], to name just a few amongst numerous others (see, for example, [12] for a recent survey).

To support these applications, much research activity has focused on developing efficient and easily implementable algorithms for their deployment $[2, \mathbf{1 7}, \mathbf{1 3}]$. One of the fastest algorithms for pairing computation on elliptic curves is that of Duursma and Lee [13], which applies to the class of supersingular elliptic curves in characteristic three with socalled embedding degree six, and is preferable in pairing implementations for contemporary security parameters.

One is therefore free to use the trace-based methods found in LUC [49] and XTR [33] for post-pairing arithmetic, resulting in the compression of pairing outputs by a factor of two and three respectively; this was pointed out to us by E. Verheul in a personal comunication. Scott and Barreto [46] also describe the use of traces for the computation of the pairing itself; however, closer inspection of their work shows that their claim is misleading. Indeed, their method is essentially a polynomial basis transformation, and hence does not offer any advantages during the computation of the pairing. Moreover, for characteristic three, we demonstrate that (contrary to the claims of Scott and Barreto [46]) the performance of their approach is inferior to a straightforward implementation. Thus, besides pairing compression, the method that they advocate does not seem to offer any benefits.

Our contribution is to achieve both efficient pairing arithmetic, and also pairing compression. Our methods are based on the simple observation that the quotient group to which the natural output of the Tate pairing belongs, may be viewed as a special representation of an algebraic torus. These groups were introduced to cryptography by Rubin and Silverberg [42], who showed under certain conditions that one can represent elements of the torus via rational embeddings into affine space, providing smaller bandwidth requirements than the corresponding field-embedded representation. 
Using this property and an efficient point-multiplication method developed for tori [25], we are able to perform arithmetic with pairing values that is on average $30 \%$ faster than previous methods. This is useful, for example, in pairing-based protocols where one typically blinds a point by an ephemeral random value. By bilinearity, this blinding may be performed either on the curve before the pairing evaluation, or in the extension field afterwards. Given that a pairing evaluation is usually several times more costly than either a point multiplication on the curve or an exponentiation in the field, if a pairing value ever needs to be re-used, it is beneficial to compute it once and for all, and to perform each ephemeral blinding in the extension field.

Examples where this occurs include the the Boneh-Franklin identity-based encryption scheme [5], the identity-based signature scheme of Hess [28], and the certificate-based encryption scheme of Gentry [21].

The aforementioned compression methods can also be used during any interactive pairingbased protocol where pairing values are transmitted between parties. Such schemes include the selective-ID identity-based encryption scheme of Boneh and Boyen [3], the interactive proof of knowledge in the short group signature scheme of Boneh et al. [4], and various others $[\mathbf{2 2}, \mathbf{4 5}]$.

One may regard our methods as a characteristic-three version of previous work on tori $[42,25]$, tailored for pairings. However, they may also be used for pairings on any abelian variety possessing an even embedding degree, which for efficiency reasons is the case for all contemporary pairing algorithms. As such, they may also be applied to supersingular binary elliptic curves, although we do not pursue this application here, since pairings based on these curves possess an inferior security/efficiency trade-off [16].

The remainder of the paper is organised as follows. We next give some background on the Tate pairing and algebraic tori. In Section 3 we develop fast arithmetic for pairing values, and in Section 4 we give algorithms for efficient exponentiation. In Section 5, we describe the field representation that we use, while in Section 6 we detail our improvements to the Duursma-Lee algorithm. In Section 7, we present implementation results, and in the final section we make some concluding remarks and present some open problems.

\section{Preliminaries}

In this section we briefly provide some mathematical background, and fix some notation.

\subsection{The Tate pairing}

The Tate pairing on an elliptic curve is usually computed using a variant of Miller's algorithm [36]. For the special curves often used in cryptography, however, it was shown independently by Barreto et al. [2] and Galbraith et al. [17] that much of the computation of the algorithm is redundant. In terms of performance, the former paper provides the better alternative, and we refer to their algorithm as the reduced Tate pairing, or the BKLS algorithm.

\subsubsection{The reduced Tate pairing}

Let $E$ be an elliptic curve over a finite field $\mathbb{F}_{q}$, and let $\mathcal{O}_{E}$ denote the identity element of the associated group of rational points on $E\left(\mathbb{F}_{q}\right)$. For a positive integer $l \mid \# E\left(\mathbb{F}_{q}\right)$ coprime to $q$, let $\mathbb{F}_{q^{k}}$ be the smallest extension field of $\mathbb{F}_{q}$ which contains the $l$ th roots of unity in $\overline{\mathbb{F}}_{q}$. Also, let $E\left(\mathbb{F}_{q}\right)[l]$ denote the subgroup of $E\left(\mathbb{F}_{q}\right)$ of all points of order dividing $l$, 
and similarly for the degree- $k$ extension of $\mathbb{F}_{q}$. From an efficiency perspective, $k$ is usually chosen to be even [2]. For a thorough treatment of the following, we refer the reader to [2] and also [17], and to [48] for an introduction to divisors. Then, assuming that $l^{2} \nmid \# E\left(\mathbb{F}_{q^{k}}\right)$, the reduced Tate pairing of order $l$ is the map

$$
e_{l}: E\left(\mathbb{F}_{q}\right)[l] \times E\left(\mathbb{F}_{q^{k}}\right)[l] \rightarrow \mathbb{F}_{q^{k}}^{\times} /\left(\mathbb{F}_{q^{k}}^{\times}\right)^{l},
$$

given by $e_{l}(P, Q)=f_{P, l}(\mathcal{D})$. Here $f_{P, l}$ is a function on $E$ whose divisor is equivalent to $l(P)-l\left(\mathcal{O}_{E}\right), \mathscr{D}$ is a divisor equivalent to $(Q)-\left(\mathcal{O}_{E}\right)$, whose support is disjoint from the support of $f_{P, l}$, and $f_{P, l}(\mathscr{D})=\prod_{i} f_{P, l}\left(P_{i}\right)^{a_{i}}$, where $\mathscr{D}=\sum_{i} a_{i} P_{i}$. It satisfies the following properties [15].

- For each $P \neq \mathcal{O}_{E}$ there exists $Q \in E\left(\mathbb{F}_{q^{k}}\right)[l]$ such that $e_{l}(P, Q) \neq 1 \in \mathbb{F}_{q^{k}}^{\times} /\left(\mathbb{F}_{q^{k}}^{\times}\right)^{l}$ (non-degeneracy).

- For any integer $n, e_{l}([n] P, Q)=e_{l}(P,[n] Q)=e_{l}(P, Q)^{n}$ for all $P \in E\left(\mathbb{F}_{q}\right)[l]$ and $Q \in E\left(\mathbb{F}_{q^{k}}\right)[l]$ (bilinearity).

- Let $L=h l$. Then $e_{l}(P, Q)^{\left(q^{k}-1\right) / l}=e_{L}(P, Q)^{\left(q^{k}-1\right) / L}$.

When one computes $f_{P, l}(\mathscr{D})$, the value obtained belongs to the quotient group $\mathbb{F}_{q^{k}}^{\times} /\left(\mathbb{F}_{q^{k}}^{\times}\right)^{l}$, and not $\mathbb{F}_{q^{k}}^{\times}$. In this quotient, for $a$ and $b$ in $\mathbb{F}_{q^{k}}^{\times}, a \sim b$ if and only if there exists $c \in \mathbb{F}_{q^{k}}^{\times}$such that $a=b c^{l}$. Clearly, this is equivalent to saying that

$$
a \sim b \text { if and only if } a^{\left(q^{k}-1\right) / l}=b^{\left(q^{k}-1\right) / l},
$$

and hence one ordinarily uses this value as the canonical representative of each coset. The isomorphism between $\mathbb{F}_{q^{k}}^{\times} /\left(\mathbb{F}_{q^{k}}^{\times}\right)^{l}$ and the elements of order $l$ in $\mathbb{F}_{q^{k}}^{\times}$given by this exponentiation makes it possible to compute $f_{P, l}(Q)$ rather than $f_{P, l}(\mathscr{D})$; see [2]. It also removes the need to compute the costly denominators in Miller's algorithm.

\subsubsection{The modified Tate pairing}

At Asiacrypt 2003, Duursma and Lee introduced an algorithm for pairing computation on a special family of supersingular hyperelliptic curves [13]. In common with the authors of [46], for the elliptic case, which occurs only in characteristic three, we refer to the algorithm as the modified Tate pairing. In Table 1, we list a sample of curves from this family, upon which we base our implementation.

Table 1: Field definitions and curve equations

\begin{tabular}{l|cccc}
\hline Field & Field polynomial & Curve & Order & MOV security \\
\hline $\mathbb{F}_{3^{79}}$ & $t^{79}+t^{26}+2$ & $Y^{2}=X^{3}-X-1$ & $3^{79}+3^{40}+1$ & 750 \\
$\mathbb{F}_{3^{97}}$ & $t^{97}+t^{12}+2$ & $Y^{2}=X^{3}-X+1$ & $\left(3^{97}+3^{49}+1\right) / 7$ & 906 \\
$\mathbb{F}_{3^{163}}$ & $t^{163}+t^{80}+2$ & $Y^{2}=X^{3}-X-1$ & $3^{163}+3^{82}+1$ & 1548 \\
$\mathbb{F}_{3^{193}}$ & $t^{193}+t^{12}+2$ & $Y^{2}=X^{3}-X-1$ & $3^{193}-3^{97}+1$ & 1830 \\
$\mathbb{F}_{3^{239}}$ & $t^{239}+t^{24}+2$ & $Y^{2}=X^{3}-X-1$ & $3^{239}-3^{120}+1$ & 2268 \\
$\mathbb{F}_{3^{353}}$ & $t^{353}+t^{142}+2$ & $Y^{2}=X^{3}-X-1$ & $3^{353}+3^{177}+1$ & 3354 \\
\hline
\end{tabular}


The first column gives the field over which each curve is defined, and the second lists the corresponding irreducible polynomials defining the field extensions. The third column lists the curve equations, and the fourth gives the order of the subgroup used. The final column gives the bit-length of the smallest finite field into which the pairing value embeds, which is a degree-six extension for these curves. These parameter values were generated simply by testing which prime extension degrees yielded orders for supersingular curves that are prime, or almost prime: that is, those possessing a small cofactor.

The modified Tate pairing improves upon the reduced variant in three ways. Firstly, by using the third property listed above instead of computing the Tate pairing of order $l$, one uses the pairing of order $q^{3}+1$, which eliminates the need for any point additions in Miller's algorithm. Secondly, while this apparently increases the trit-length of the exponent by a factor of three (where a trit is a ternary digit, by analogy with 'bit'), Duursma and Lee show that the divisor computed when processing three trits at a time has a very simple form, and hence no losses are incurred. Lastly, they provide a closed form expression for the pairing, thus simplifying implementations. We give a full description of the Duursma-Lee algorithm in Section 6, where we also make some elementary computational improvements.

\subsection{Algebraic tori}

In 1985, ElGamal [14] made the suggestion that Diffie-Hellman key exchange, digital signatures and ElGamal encryption be performed in the multiplicative group of an extension of $\mathbb{F}_{p}$, although without going into details. Recent trends in cryptographic research have shown that by exploiting the algebraic structure not available in prime fields, one can obtain compression of elements and efficient arithmetic.

Due to the observation of Pohlig and Hellman [39], one typically works in a prime-order subgroup of sufficient size in the multiplicative group of the extension field. To ensure that a particular subgroup does not embed into any subfield of the extension field, it must belong to the cyclotomic subgroup [32], which conjecturally attains the discrete logarithm security of the extension field. The public key cryptosystem XTR [33] exploits compression of elements in the cyclotomic subgroup of $\mathbb{F}_{p^{6}}^{\times}$by taking their trace with respect to the quadratic subfield, to obtain a compression factor of three.

Based on similar ideas, Rubin and Silverberg [42] proposed the notion of torus-based cryptography as an alternative way to obtain compression of elements in the cyclotomic subgroup of a suitable field extension, which is isomorphic to an algebraic torus (cf. Lemma 1). The public key system CEILIDH proposed in that paper is based on the torus $T_{6}$. This torus has the property that it is birationally isomorphic to two-dimensional affine space, which means that its elements can be parametrised via rational functions by only two elements of the base field, rather than the six elements ordinarily required.

It was also shown in [42] that efforts to find a natural extension of the trace-based method of XTR using symmetric functions [7] cannot work. It is an open conjecture whether or not $T_{n}$ is 'rational' for all $n$, in which case one could efficiently compress elements of $T_{n}$ by a factor of $n / \phi(n)$; see $[\mathbf{5 4 , 4 2}$. This conjecture is known to be true when $n$ is either a prime power, or the product of two prime powers. However, for the applications that concern us here, the status of the conjecture is unlikely to have any impact, as we explain in Section 6.

\subsubsection{The torus $T_{n}\left(\mathbb{F}_{q}\right)$}

Let $\mathbb{F}_{q}$ be a finite field where $q$ is a power of a prime, and let $\Phi_{n}$ be the $n$th cyclotomic polynomial. We write $G_{q, n}$ for the subgroup of $\mathbb{F}_{q^{n}}^{\times}$of order $\Phi_{n}(q)$, and let $\mathbb{A}^{n}\left(\mathbb{F}_{q}\right)$ denote the $n$-dimensional affine space over $\mathbb{F}_{q}$ : that is, the variety whose points lie in $\mathbb{F}_{q}^{n}$. 
Definition 1. Let $k=\mathbb{F}_{q}$ and $L=\mathbb{F}_{q^{n}}$. The torus $T_{n}$ is the intersection of the kernels of the norm maps $N_{L / F}$, for all subfields $k \subset F \subsetneq L$ :

$$
T_{n}(k):=\bigcap_{k \subset F \subsetneq L} \operatorname{Ker}\left[N_{L / F}\right] .
$$

The following lemma provides some relevant properties of $T_{n}$.
LEMMA 1 (see [42]).
(i) $T_{n}\left(\mathbb{F}_{q}\right) \cong G_{q, n}$, and thus $\# T_{n}\left(\mathbb{F}_{q}\right)=\Phi_{n}(q)$.

(ii) If $h \in T_{n}\left(\mathbb{F}_{q}\right)$ is an element of prime order not dividing $n$, then $h$ does not lie in a proper subfield of $\mathbb{F}_{q^{n}} / \mathbb{F}_{q}$.

\section{The quotient group}

Throughout this section and the remainder of the paper we assume that we are working in characteristic-three fields with prime extension degree (though the ideas apply equally well to arbitrary finite fields, with some minor deviations for the binary case) and so, where relevant, all exponents are written in ternary.

Let $l \mid \# E\left(\mathbb{F}_{q}\right)$ and suppose that we wish to compute the modified Tate pairing of order $l$. Then, invoking the third property of Section 2.1, one uses the Duursma-Lee algorithm to first compute $e_{q^{3}+1}$, which is an element in the quotient group

$$
g=\mathbb{F}_{q^{6}}^{\times} /\left(\mathbb{F}_{q^{6}}^{\times}\right)^{q^{3}+1}
$$

For any $a \in \mathbb{F}_{q^{6}}^{\times}$we have $a^{q^{3}+1} \in \mathbb{F}_{q^{3}}^{\times}$, and so $g$ simplifies to $\mathbb{F}_{q^{6}}^{\times} / \mathbb{F}_{q^{3}}^{\times}$.

Let $G_{l} \subset \mathbb{F}_{q^{6}}^{\times}$denote the subgroup of order $l$, and let $e \in \mathcal{g}$. Then the two properties:

$$
\operatorname{gcd}\left(l, q^{3}-1\right)=1 \quad \text { and } \quad e^{q^{3}-1} \in G_{l}
$$

imply that $e=g h$ for some $g \in G_{l}, h \in \mathbb{F}_{q^{3}}^{\times}$. Hence powering $e$ by $q^{3}-1$ gives

$$
e^{q^{3}-1}=(g h)^{q^{3}-1}=g^{q^{3}-1},
$$

which can then be used in protocols. If a particular protocol requires an exponentiation of this value by some integer $k \bmod l$, this is performed in $\mathbb{F}_{q^{6}}$.

In this section we give an alternative way to obtain unique representatives of $g$ easily, that furthermore permits fast multiplication, and provides automatic compression by a factor of two. We then show that the natural embedding of $g$ into the extension field is just a special representation of an algebraic torus, which permits further compression.

\subsection{The basic idea}

Let $\mathbb{F}_{q^{6}}=\mathbb{F}_{q^{3}}[\sigma] /\left(\sigma^{2}+1\right)$, which is the extension that we use in the Duursma-Lee algorithm. Writing $e=e_{0}+e_{1} \sigma$ and $g=g_{0}+g_{1} \sigma$, by the above we have

$$
e=g h=g_{0} h+g_{1} h \sigma .
$$

Since the represented coset remains invariant under multiplication by elements of $\mathbb{F}^{\times} q^{3}$, we can divide by $e_{1}$, giving

$$
e^{\prime}=e e_{1}^{-1}=e_{0} / e_{1}+\sigma=g_{0} / g_{1}+\sigma .
$$

This also eliminates $h$, and may equally well be used as a canonical representative of the coset to which $e$ belongs. 
This element of the quotient group can be represented simply by the $\mathbb{F}_{q}$ element $e_{0} / e_{1}$, and thus compresses the coset representation by a factor of two. Computationally, this involves a division in $\mathbb{F}_{q^{3}}$.

Comparing this to powering by $q^{3}-1$, the saving is not significant, since:

$$
e^{q^{3}-1}=\frac{e_{0}-e_{1} \sigma}{e_{0}+e_{1} \sigma}
$$

and hence requires only a division in $\mathbb{F}_{q^{6}}$.

However, if one exponentiates this value by some integer $k \bmod l$, this operation will be faster than if one had first powered $e$ by $q^{3}-1$, since multiplying a generic element of $g$ by this element is cheaper than multiplying two generic elements. To see this, let $g=g_{0} / g_{1}=e_{0} / e_{1}$ and $a_{0}+a_{1} \sigma \in g$. Then

$$
(g+\sigma)\left(a_{0}+a_{1} \sigma\right)=\left(g a_{0}-a_{1}\right)+\left(g a_{1}+a_{0}\right) \sigma,
$$

which costs just two $\mathbb{F}_{q^{3}}$ multiplications, and not the three required if both elements are generic, in which case the arithmetic is identical to that of $\mathbb{F}_{q^{6}}$. If one assumes that cubings and additions are essentially free, then this method will always be roughly one third faster, for whatever practical method one uses to exponentiate. The defining property of the quotient group $g$ thus reduces the cost of arithmetic performed on pairing values.

\subsection{Arithmetic in $g$}

We first introduce some terminology to clarify the operations available in $g$. The property that a given coset is invariant under multiplication by elements of $\mathbb{F}_{q^{3}}^{\times}$is suggestive of the projective line

$$
\mathbb{P}^{1}\left(\mathbb{F}_{q^{3}}\right)=\left\{(x, y) \in\left(\mathbb{F}_{q^{3}}\right)^{2} \backslash\{(0,0)\}\right\} / \sim
$$

where $\left(x_{1}, y_{1}\right) \sim\left(x_{2}, y_{2}\right)$ if and only if a $\lambda \in \mathbb{F}_{q^{3}}^{\times}$exists such that $\left(x_{1}, y_{1}\right)=\left(\lambda x_{2}, \lambda y_{2}\right)$. The reduction of $e$ to $e_{0} / e_{1}$ may also be viewed as a map to the affine line $\mathbb{A}^{1}\left(\mathbb{F}_{q^{3}}\right)$. With this analogy, we introduce the following definition.

DEFINITION 2. $g_{P}$ is the projective line $\mathbb{P}^{1}\left(\mathbb{F}_{q^{3}}\right)$ endowed with the group operation induced by the arithmetic of the quadratic extension $\mathbb{F}_{q^{6}}=\mathbb{F}_{q^{3}}[\sigma] /\left(\sigma^{2}+1\right)$ via the map $(x, y) \rightarrow$ $x+y \sigma$. The identity element is represented by the point $(\lambda, 0)$ for any $\lambda \in \mathbb{F}_{q^{3}}$.

$g_{A}$ is the affine part of the line $g_{P}$. The affine point corresponding to $(x, y)$ is $X=$ $A(x, y)=(x / y)$. Via this map, the identity element is the point at infinity which we denote by $\mathcal{O}_{g}$.

With this terminology, it should be clear that we can mimic mixed addition methods for point multiplication on elliptic curves [9]. The use of signed digit representations follows since inverses are cheap, as we show below. In Section 4 we derive an exponentiation algorithm using a split exponent method.

Let $P=(x, y) \in g_{P}$ with corresponding affine representation $(X) \in g_{A}$. We refer to the generator of $\operatorname{Gal}\left(\mathbb{F}_{q^{6}} / \mathbb{F}_{q}\right)$ as the $q$-Frobenius (that is, the automorphism given by powering by $q$ ). As already stated, computing the inverse of an element is virtually free. This follows since the order of $g$ is

$$
\left|\mathbb{F}_{q^{6}}^{\times} / \mathbb{F}_{q^{3}}^{\times}\right|=\left(q^{6}-1\right) /\left(q^{3}-1\right)=q^{3}+1,
$$

and so applying the cube of the Frobenius gives the inverse: $P^{-1}=(x,-y)$ or $(-X)$ in affine. Cubing is also straightforward, since we are working in characteristic three: $P^{3}=$ $\left(x^{3},-y^{3}\right)$. 
For multiplication of two points $P_{1}=\left(x_{1}, y_{1}\right), P_{2}=\left(x_{2}, y_{2}\right) \in g_{P}$ with affine representations $\left(X_{1}\right),\left(X_{2}\right) \in g_{A}$, we use the following easy lemma.

LEMMA 2. Let $M$ and I represent the cost of a multiplication and an inversion, respectively, in $\mathbb{F}_{q}$. Then the group operation for combinations of point representations is computed as shown in Table 2.

Table 2: The group operation for combinations of point representations

\begin{tabular}{ccccc}
\hline$P_{1}$ & $P_{2}$ & $P_{1} \cdot P_{2}$ & Formula & Cost \\
\hline$g_{A}$ & $g_{A}$ & $g_{A}$ & $\left(X_{1} X_{2}-1\right) /\left(X_{1}+X_{2}\right)$ & $2 M+I$ \\
$g_{A}$ & $g_{A}$ & $g_{P}$ & $\left(X_{1} X_{2}-1, X_{1}+X_{2}\right)$ & $1 M$ \\
$g_{P}$ & $g_{P}$ & $g_{A}$ & $\left(x_{1} x_{2}-y_{1} y_{2}\right) /\left(x_{1} y_{2}+x_{2} y_{1}\right)$ & $4 M+I$ \\
$g_{P}$ & $g_{P}$ & $g_{P}$ & $\left(x_{1} x_{2}-y_{1} y_{2}, x_{1} y_{2}+x_{2} y_{1}\right)$ & $3 M$ \\
$g_{A}$ & $g_{P}$ & $g_{A}$ & $\left(X_{1} x_{2}-y_{2}\right) /\left(X_{1} y_{2}+x_{2}\right)$ & $3 M+I$ \\
$g_{A}$ & $g_{P}$ & $g_{P}$ & $\left(X_{1} x_{2}-y_{2}, X_{1} y_{2}+x_{2}\right)$ & $2 M$ \\
\hline
\end{tabular}

Squaring can, naturally, be performed with slightly fewer $\mathbb{F}_{q^{3}}$ muliplications than above; the corresponding formulae are easily deduced. Besides the precomputation necessary for the exponentiation algorithms that we present in Section 4, however, squarings are not required.

With regard to exponentiations, it is clear that the mixed multiplication shown in the final row is the most efficient. If we want to compute $P^{k}$ for some $k \bmod l$, we first convert $P$ to affine, and for each non-zero trit in the expansion of $k$ we perform a mixed multiplication of this point with the projective representation of the intermediate value. A multiplication with both points in projective form is equivalent to an ordinary multiplication in $\mathbb{F} q^{6}$, so the mixed multiplication is essentially what allows the savings over arithmetic in $\mathbb{F} q^{6}$. We exploit these observations in the exponentiation algorithms developed in Section 4.

\subsection{An equivalent representation of the quotient group}

The arithmetic just described for the quotient group is essentially identical to that developed for the torus $T_{2}$; see $[25,42]$. Indeed, it is not difficult to see that $g$ is isomorphic to $T_{2}\left(\mathbb{F}_{q^{3}}\right)$, and that the affine arithmetic for $g$ is identical to that given by Rubin and Silverberg for the compressed representation of $T_{2}\left(\mathbb{F}_{q^{3}}\right)$.

Given $e \in \mathcal{g}$, it is possible to compute the embedding $e^{q^{3}} / e$ of $e$ into $\mathbb{F}_{q^{6}}$ and maintain invariance under multiplication by elements of $\mathbb{F}_{q^{3}}^{\times}$. This may seem odd, since $\mathbb{F}_{q^{6}}$ does not possess this property. However, our choice of representation of elements in the subgroup of order $q^{3}+1$ makes this possible. Again, let $e=e_{0}+e_{1} \sigma$. Then

$$
e^{q^{3}}=e_{0}-e_{1} \sigma
$$

and hence

$$
e^{q^{3}-1}=\frac{e_{0}-e_{1} \sigma}{e_{0}+e_{1} \sigma} \in G_{l} \subset \mathbb{F}_{q^{6}}
$$

One can perform this division in $\mathbb{F}_{q^{6}}$ and use the ordinary polynomial representation. Here we choose to leave this fraction unevaluated. Note that multiplying the numerator and denominator of (1) by any element of $\mathbb{F}_{q^{3}}^{\times}$leaves the represented element unchanged. 
An interesting property of this representation is that when multiplying two fractions of this form, the coefficients of the numerator and the denominator correspond exactly: let

$$
c=\frac{c_{0}-c_{1} \sigma}{c_{0}+c_{1} \sigma}, \quad d=\frac{d_{0}-d_{1} \sigma}{d_{0}+d_{1} \sigma},
$$

with $c_{i}, d_{i} \in \mathbb{F}_{q^{3}}$. Then, since $\sigma^{2}+1=0$, we see that

$$
c d=\frac{\left(c_{0} d_{0}-c_{1} d_{1}\right)-\left(c_{0} d_{1}+c_{1} d_{0}\right) \sigma}{\left(c_{0} d_{0}-c_{1} d_{1}\right)+\left(c_{0} d_{1}+c_{1} d_{0}\right) \sigma} .
$$

This also follows trivially from the fact that $(c d)^{q^{3}-1}=c^{q^{3}-1} \cdot d^{q^{3}-1}$.

For an implementation, this allows one therefore to work with the denominator only, since one knows that the coefficients of the numerator will be identical. Hence one may view our previous operations in $g$ without powering equivalently as operating purely on the denominator of (1) after powering, and so all the arithmetic carries over unchanged.

REMARK 1. The representation (1) and its identification with $\mathbb{P}^{1}$ were given explicitly by Rubin and Silverberg [42]. However, the arithmetical consequences of embedding this representation into the extension field were only fully considered in [25], where it was also noted that one may also represent $T_{2}$ as a quotient group. Thus while these ideas are not new (the representation (1) is a simple application of Hilbert's Theorem 90), they find a novel application in pairing-based cryptography.

\subsection{Further compression using $T_{6}\left(\mathbb{F}_{q}\right)$}

Since the characteristic-three supersingular elliptic curves that we consider have embedding degree six, one may ask why we use the arithmetic of $T_{2}\left(\mathbb{F}_{q^{3}}\right)$ when the order- $l$ subgroup is in fact in $T_{6}\left(\mathbb{F}_{q}\right)$. The reason is that there seems no obvious way to utilise the extra structure provided by $T_{6}\left(\mathbb{F}_{q}\right.$ ) (see [25]), though we do not rule out such a possibility. However, we know that $\left|T_{6}\left(\mathbb{F}_{q}\right)\right|=\left(q^{2}-q+1\right)\left|\left(q^{3}+1\right)=\right| T_{2}\left(\mathbb{F}_{q^{3}}\right) \mid$, and so $T_{6}\left(\mathbb{F}_{q}\right) \subset T_{2}\left(\mathbb{F}_{q^{3}}\right)$. Thus one can use the properties of the latter and apply them to the former, utilising the improvements derived over the extension field representation.

While arithmetic improvements do not seem to be available with $T_{6}$, one can exploit it for better compression. As $T_{6}$ is rational, one can map nearly all its elements to the affine plane and use this representation instead for data transmissions.

Using the method described by Rubin and Silverberg [42], and thanks to some serendipitous equations for characteristic three and the given field representation, one obtains this additional compression for free.

By Definition 1,

$$
T_{6}\left(\mathbb{F}_{q}\right)=\operatorname{Ker}\left(N_{\mathbb{F}_{q^{6}} / \mathbb{F}_{q^{3}}}\right) \cap \operatorname{Ker}\left(N_{\mathbb{F}_{q^{6}} / \mathbb{F}_{q^{2}}}\right)=T_{2}\left(\mathbb{F}_{q^{3}}\right) \cap \operatorname{Ker}\left(N_{\mathbb{F}_{q^{6}} / \mathbb{F}_{q^{2}}}\right) .
$$

To obtain a suitable representation, one therefore needs only to parametrise those elements of the form (1) which have norm equal to one in the second factor.

Let $e=(a-\sigma) /(a+\sigma)$ be the compressed representation for $e$, and let $a=$ $a_{0}+a_{1} \rho+a_{2} \rho^{2}$, where $\rho^{3}-\rho \pm 1=0$ defines the cubic extension that we later use for the Duursma-Lee algorithm. Then we obtain an equation in $a_{0}, a_{1}$, and $a_{2}$ by the condition

$$
\left(\frac{a-\sigma}{a+\sigma}\right)^{1+q^{2}+q^{4}}=1 \text {. }
$$


This is equivalent to $1+a_{1}^{2}-a_{0} a_{2}-a_{2}^{2}=0$, which one can parametrise easily with just $a_{1}$ and $a_{2}$, since $a_{0}=\left(1+a_{1}^{2}-a_{2}^{2}\right) / a_{2}$. It is therefore sufficient to specify only $a_{1}$ and $a_{2}$, to describe all points on $T_{6}\left(\mathbb{F}_{q}\right)$ bar the identity, and this is essentially all that we need. We therefore have a map

given by

$$
\psi: \mathbb{A}^{2}\left(\mathbb{F}_{q}\right) \backslash\left\{\left(a_{1}, 0\right)\right\} \rightarrow T_{6}\left(\mathbb{F}_{q}\right) \backslash\{1\}
$$

$$
\psi\left(a_{1}, a_{2}\right)=\frac{\left(\left(1+a_{1}^{2}-a_{2}^{2}\right)+a_{1} a_{2} \rho+a_{2}^{2} \rho^{2}\right)-a_{2} \sigma}{\left(\left(1+a_{1}^{2}-a_{2}^{2}\right)+a_{1} a_{2} \rho+a_{2}^{2} \rho^{2}\right)+a_{2} \sigma} .
$$

The inverse map

$$
\psi^{-1}: T_{6}\left(\mathbb{F}_{q}\right) \backslash\{1\} \rightarrow \mathbb{A}^{2}\left(\mathbb{F}_{q}\right) \backslash\left\{\left(a_{1}, 0\right)\right\}
$$

is given as above; that is, we just take the second and third coefficients in the fractional expression for $e$.

Note that $\mathbb{A}^{2}\left(\mathbb{F}_{q}\right) \backslash\left\{\left(a_{1}, 0\right)\right\}$ and $T_{6}\left(\mathbb{F}_{q}\right) \backslash\{1\}$ both have cardinality $q^{2}-q$. In terms of the quotient group $g$ and an actual pairing computation, once $e_{0} / e_{1}$ has been computed one can use the second and third coefficients to parametrise the element, without any further computation.

REMARK 2. In the context of compression, Rubin and Silverberg [41, 43] have shown how one can compress BLS short-signatures [6] by using the trace-zero subvariety contained in the Weil restriction of scalars of an elliptic curve defined over a composite field extension. This method provides a compression factor of $n / \phi(n)$ also, where $\operatorname{gcd}(n, 2)=1$, and can be applied to any pairing-based protocol where one is required to transmit a point on the curve, such as [29]. However for $n \geqslant 5$, building upon an idea of Semaev [47], Gaudry has shown [20] that such curves are weaker than those defined over the prime field, and hence this method should be regarded with some caution. We point out that this form of precompression is distinct from the post-compression described here, and thus these attacks do not apply.

\section{Exponentiation}

Now that we have set up the basic arithmetic for the quotient group $g=\mathbb{F}^{\times} /\left(\mathbb{F}_{q^{6}}^{\times}\right)^{q^{3}+1}$, we explore how one can optimise the basic operation of exponentiation in practice. For comparison, we also describe fast algorithms for exponentation in the order- $l$ subgroup $G_{l} \subset \mathbb{F}_{q^{6}}^{\times}$using existing techniques [52] and point multiplication in $E\left(\mathbb{F}_{q}\right)$, incorporating a novel technique that we develop here.

For ease of notation, we write the group operation for all three groups multiplicatively, and for each of the above we compare four exponentiation methods, which we detail in turn. The input to each algorithm is a base $e$ and an integer $k \bmod l$ in standard ternary format. The output is $e^{k}$. When applicable, precomputed values are stored in affine to facilitate the mixed multiplication. We note that in all three groups, inversions are essentially for free, so we consider signed digit representations. For the remainder of the paper, $m$ represents the cost of one $\mathbb{F}_{q}$ multiplication.

\section{Method 1: Signed ternary expansion}

Using the generalised non-adjacent form, or G-NAF [8], one can take the ternary expansion of an exponent $k \bmod l$ and transform it into an equivalent signed ternary representation. Such a representation is easy to compute, and reduces the average density of non-zero trits from two thirds to one half. The precomputation involves just a single squaring of the base. 


\section{Method 2: Signed nonary expansion}

This is the same as Method 1, except we use a base-nine expansion of $k$. This essentially halves the trit-length of $k$ for the cost of precomputing $e^{i}, i=1, \ldots, 8$. Again using the G-NAF, the average density of non-zero 'nits' in this expansion is four fifths.

\section{Method 3: Sliding window ternary expansion}

We use an unsigned ternary expansion of $k$ with a sliding window of width three [35, Chapter 14, Algorithm 14.85]. To do so, one needs to precompute and store $e^{i}$ for $0<i<27$ and $i \neq 0 \bmod 3$.

\section{Method 4: Frobenius expansion}

For $e \in g$, the $q$-Frobenius map is easily computed. Moreover, the $q$ th power of a compressed element is itself compressed. Since the Frobenius map satisfies $q^{2}-q+1=0$ (as maps) and the group order divides $q^{2}-q+1$, one can split the exponent $k$ in two halves $k_{1}$ and $k_{2}$, where $k_{1}, k_{2}$ are approximately half the trit-length of $l$ and satisfy $k \equiv$ $k_{1}+k_{2} q \bmod l$; see [52]. One can find $k_{1}$ and $k_{2}$ very quickly, having performed a one-time Gaussian two-dimensional lattice basis reduction.

Thus a single exponentiation can be transformed into a double exponentiation for half the trit-length of $k$, for the cost of performing a double exponentiation instead. To compute $e^{k}$ for a random $k$ mod $l$, we perform the double exponentiation $e^{k_{1}}\left(e^{q}\right)^{k_{2}}$ using Shamir's trick, originally due to Straus [53]. We detail the required precomputation in the next section.

For each of $k_{1}, k_{2}$ we invoke the G-NAF. The average density of non-zero trits in each of their ternary expansions is $1 / 2$, and hence the average number of non-zero trits in the paired ternary expansion of $k_{1}, k_{2}$ is $1-(1 / 2)^{2}=3 / 4$. We therefore expect to perform on average $(3 / 4) \cdot m / 2=(3 / 8) m$ multiplications of mixed type during an exponentiation.

Interestingly enough, this method also works for the elliptic curve. Clearly, one can use the same expansion of $k$ on $E\left(\mathbb{F}_{q}\right)$, with powering by $q$ replaced by scalar multiplication by $q$. Somewhat surprisingly, on the curve also, multiplication by $q$ is an efficiently computable automorphism since $[q] P=(x-(m \bmod 3) b,-y)$ for $P=(x, y)$ on the curve (where the curve equation is $Y^{2}=X^{3}-X+b$ ). Thus we arrive at a novel application of the Gallant-Lambert-Vanstone exponent split method using fast automorphisms [18].

We note that for supersingular curves over characteristic three, there is also an efficient scalar multiplication algorithm due to Koblitz [30] based on the curve automorphism mapping the point $(x, y)$ to $\left(x^{3}, y^{3}\right)$.

\subsection{Precomputation}

The necessary precomputation for Methods 1, 2 and 3 is straightforward. For Method 4, we can take advantage of the $q$-Frobenius to reduce the cost. We use the notation of $g$. Let $e=e_{0}+e_{1} \sigma$. In order to use Shamir's trick, we need to know the values

$$
\left(e_{0} / e_{1}+\sigma\right)^{i+q j} \quad i, j \in\{0, \pm 1, \pm 2\}
$$

in affine. Let $(i, j)$ represent the corresponding term in (2). Then we can use the fact that for any $e \in \mathcal{g}$, we have $e^{q^{2}-q+1}=\mathcal{O}_{g}$ to generate most of the required terms easily. To achieve this, one applies the $q$-Frobenius iteratively to obtain $(i, j)^{q}=(-j, i+j)$. We list these operations in Algorithm 1. In $G_{l}$ we use the same method, having first powered $e$ by $q^{3}-1$, but clearly without needing to obtain affine representatives. 


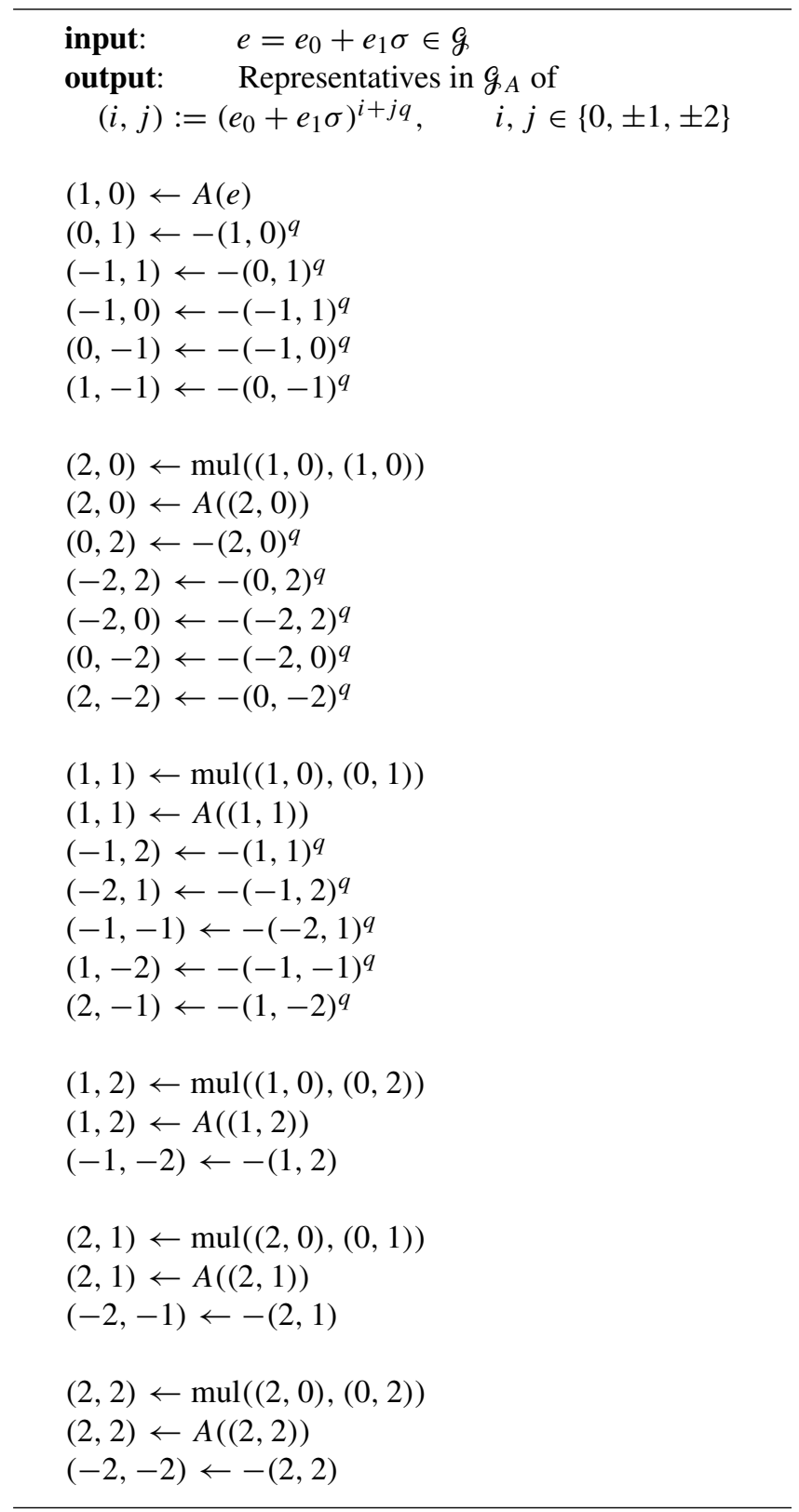

Algorithm 1: Online pre-computation for double exponentiation

\subsection{Comparison with trace-based exponentiation}

The cost of a mixed multiplication in $g$ is $12 M$. Since $l \approx 3^{m}$, an exponentiation using Method 4 costs on average about $4.5 \mathrm{mM}$. This improves considerably on the $12 \mathrm{mM}$ required by the trace method of [46]. Even without mixed multiplication, this exponentiation still 
only requires $6.75 \mathrm{~m} \mathrm{M}$, and with neither the exponent splitting nor the mixed multiplication, this cost is only about $9 m M$. Hence ordinary field arithmetic outperforms the proposed trace method, which can in fact be reduced further to about $10.3 m M$ using a Euclidean algorithm [51], but is still over twice as slow.

\subsection{Application to other pairings}

We have focused primarily on small characteristic tori because the Duursma-Lee algorithm is currently ${ }^{1}$ the most efficient for pairing computation. In the future, the preferred embedding degree of a curve will increase in order to maintain a good security/efficiency trade-off, and thus it is likely that non-supersingular curves over large characteristic fields will be used.

Since the embedding degree $n$ of a pairing on a given abelian variety is minimal, the output of any pairing may be considered an element of the torus $T_{n}$. Hence all of the techniques developed for torus-based cryptography may be applied, certainly for any embedding degree less than thirty.

However, earlier work [25] shows that for large characteristic, the trace-based methods such as LUC [49] (for degree-two extensions), and XTR [33, 51] (for degree-six extensions), are slightly faster than the torus approach. For the near future, however, our methods are likely to remain near-optimal.

\section{Field representation}

We briefly describe efficient arithmetic for $\mathbb{F}_{q}$ and the required extensions.

\subsection{Field arithmetic in $\mathbb{F}_{q}$}

Let $\mathbb{F}_{q}=\mathbb{F}_{3^{m}}$. Let $a=a_{m-1} x^{m-1}+\ldots+a_{1} x+a_{0}$ be an element of $\mathbb{F}_{q}$, held in a polynomial basis, so that $a_{i} \in \mathbb{F}_{3}$. We follow other work $[17,27]$ and represent the element $a$ as two bit-vectors $a_{H}$ and $a_{L}$. If we let $a_{H}[i]$ and $a_{L}[i]$ denote bit $i$ of $a_{H}$ and bit $i$ of $a_{L}$ respectively, the vectors $a_{H}$ and $a_{L}$ are constructed from $a$ such that for all $i$

$$
\begin{aligned}
a_{H}[i] & =a_{i} \operatorname{div} 2 ; \\
a_{L}[i] & =a_{i} \bmod 2 .
\end{aligned}
$$

That is, $a_{H}$ and $a_{L}$ are a bit-sliced representation of the coefficients of $a$ where $a_{H}$ holds the high bit and $a_{L}$ the low bit of a given coefficient. Given a representation of this type, we can perform a component-wise addition $r_{i}=a_{i}+b_{i}$ of two elements $a$ and $b$ using the following word-wise logical operations:

$$
\begin{aligned}
r_{H}[i] & =\left(a_{L}[i] \vee b_{L}[i]\right) \oplus t, \\
r_{L}[i] & =\left(a_{H}[i] \vee b_{H}[i]\right) \oplus t,
\end{aligned}
$$

where

$$
t=\left(a_{L}[i] \vee b_{H}[i]\right) \oplus\left(a_{H}[i] \vee b_{L}[i]\right)
$$

\footnotetext{
${ }^{1}$ After the initial submission of this paper and the dissemination of a preprint of it [26], generalizations of the Duursma-Lee algorithm were found [1, 31]. In particular the $\eta$-pairing [1] can be faster than the Duursma-Lee algorithm optimized in this paper. However, both our methods of exploiting the sparsity of the multiplicands (to be discussed in Section 6) and the techniques to deal with the final exponentiations and handle the resulting pairing value (described in this section) apply to these more recent results.
} 
Subtraction, and hence multiplication by two, are equally efficient since the negation of an element $a$ simply swaps the vectors $a_{H}$ and $a_{L}$ over, and can therefore be implemented by the same function as addition.

On a given computer with word-size $w$, we hold the bit-vectors $a_{H}$ and $a_{L}$ that represent $a$ as two word-vectors of length $n=\lceil m / w\rceil$, and hence we can apply logical operations in parallel to $w$ coefficients at a time. However, since our representation remains bit-oriented, we can borrow further techniques developed for fields of characteristic two. Specifically, it is possible to construct multiplication using a variation of the often-cited 'comb' method [34] and inversion by altering the binary extended Euclidean algorithm. We used a Karatsuba method to aggressively split the multiplication operands into word-sized chunks, an option that provided significant performance improvements. Unlike elements in characteristic two, squaring in characteristic three is only marginally less expensive than general multiplication. However, cubing can be performed very quickly using table-lookup in an analogous way to the so-called coefficient thinning method in characteristic two.

\subsection{Field arithmetic in $\mathbb{F}_{q^{3}}$}

Let $\mathbb{F}_{q^{3}}=\mathbb{F}_{q}[\rho] /\left(\rho^{3}-\rho-b\right)$, with $b= \pm 1$ depending on the curve equation. Let

$$
a=a_{0}+a_{1} \rho+a_{2} \rho^{2} \quad \text { and } \quad b=b_{0}+b_{1} \rho+b_{2} \rho^{2}
$$

be two generic elements. We require the following operations.

\section{$q$-Frobenius}

Since $\rho^{3}=\rho+b$, we have $\rho^{3^{m}}=\rho+(m \bmod 3) b$ and $\left(\rho^{2}\right)^{3^{m}}=\left(\rho^{3^{m}}\right)^{2}=\rho^{2}+$ $2 b(m \bmod 3) \rho+\left(m^{2} \bmod 3\right)$. Hence

$$
\begin{aligned}
a^{3^{m}} & =\left(a_{0}+a_{1} \rho+a_{2} \rho^{2}\right)^{3^{m}} \\
& =\left(a_{0}+a_{1} b(m \bmod 3)+a_{2} b\right)+\left(a_{1}-a_{2} b(m \bmod 3)\right) \rho+a_{2} \rho^{2} .
\end{aligned}
$$

\section{Multiplication}

Let $t_{00}=a_{0} b_{0}, t_{11}=a_{1} b_{1}, t_{22}=a_{2} b_{2}, t_{01}=\left(a_{0}+a_{1}\right)\left(b_{0}+b_{1}\right), t_{12}=\left(a_{1}+a_{2}\right)\left(b_{1}+b_{2}\right)$, and $t_{20}=\left(a_{2}+a_{0}\right)\left(b_{2}+b_{0}\right)$. Then

$a b=\left(t_{00}+\left(t_{12}-t_{11}-t_{22}\right) b\right)+\left(t_{01}-t_{00}+t_{11}+t_{12}+t_{22}(b-1)\right) \rho+\left(t_{20}-t_{00}+t_{11}\right) \rho^{2}$.

\section{Cubing}

This is straightforward in characteristic three, since

$$
a^{3}=\left(a_{0}^{3}+a_{2}^{3}+a_{1}^{3} b\right)+\left(a_{1}^{3}-a_{2}^{3} b\right) \rho+a_{2}^{3} \rho^{2} .
$$

\section{Inversion}

Since the extension degree is small, we can perform this directly. Let $t_{00}=a_{0}^{2}, t_{11}=a_{1}^{2}$, $t_{22}=a_{2}^{2}, t_{01}=a_{0} a_{1}, t_{12}=a_{1} a_{2}, t_{20}=a_{2} a_{0}$, and let $\Delta=a_{0}^{3}+a_{1}^{3} b+a_{2}^{3}+t_{20}\left(a_{2}-a_{0}\right)-$ $a_{1}\left(t_{01}+t_{22} b\right)$. Then

$$
a^{-1}=\Delta^{-1}\left(\left(t_{00}-t_{20}+t_{22}-t_{11}-t_{12} b\right)+\left(t_{22} b-t_{01}\right) \rho+\left(t_{11}-t_{20}-t_{22}\right) \rho^{2}\right) .
$$

\subsection{Field arithmetic in $\mathbb{F}_{q^{6}}$}

Let $\mathbb{F}_{q^{6}}=\mathbb{F}_{q^{3}}[\sigma] /\left(\sigma^{2}+1\right)$. Let $c=c_{0}+c_{1} \sigma$ and $d=d_{0}+d_{1} \sigma$ with $c_{i}, d_{i} \in \mathbb{F}_{q^{3}}$ being two generic elements. The arithmetic is as follows. 


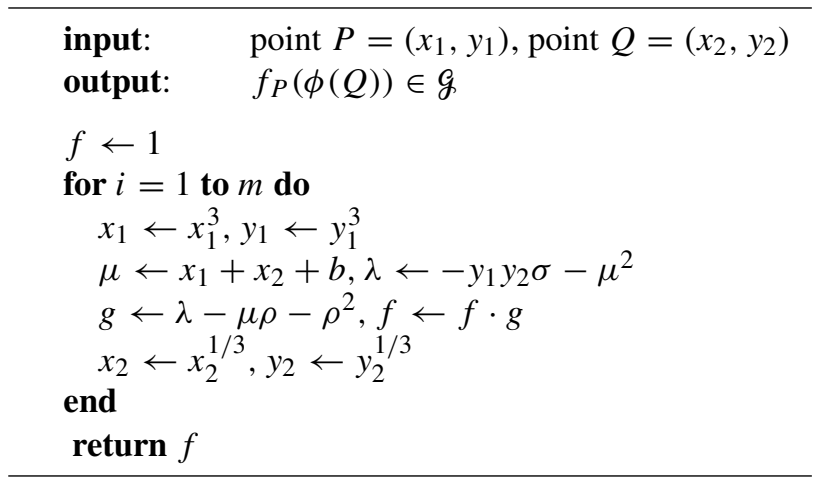

Algorithm 2: The Duursma-Lee algorithm

$q$-Frobenius

Since $\sigma^{2}=-1$, we have $\sigma^{3}=-\sigma$ and, as $m$ is odd, we obtain

$$
c^{3^{m}}=c_{0}^{3^{m}}-c_{1}^{3^{m}} \sigma \text {. }
$$

\section{Multiplication}

Let $t_{00}=c_{0} d_{0}, t_{11}=c_{1} d_{1}$, and $t_{01}=\left(c_{0}+c_{1}\right)\left(d_{0}+d_{1}\right)$. Then

$$
c d=\left(t_{00}-t_{11}\right)+\left(t_{01}-t_{00}-t_{11}\right) \sigma .
$$

Cubing

$$
c^{3}=c_{0}^{3}-c_{1}^{3} \sigma
$$

Inversion

Let $\Delta=c_{0}^{2}+c_{1}^{2}$. Then

$$
c^{-1}=\Delta^{-1}\left(c_{0}-c_{1} \sigma\right)
$$

\section{The modified Tate pairing algorithm}

In this section we detail how to efficiently implement the Duursma-Lee algorithm for the computation of the modified Tate pairing.

Let $P=\left(x_{1}, y_{1}\right)$ and $Q=\left(x_{2}, y_{2}\right)$ be points of order $l$. Then the modified Tate pairing on the supersingular curve $E\left(\mathbb{F}_{q}\right): Y^{2}=X^{3}-X+b$ is the mapping $f_{P}(\phi(Q))^{q^{3}-1}$, where $\phi: E\left(\mathbb{F}_{q}\right) \rightarrow E\left(\mathbb{F}_{q^{6}}\right)$ is the distortion map $\phi\left(x_{2}, y_{2}\right)=\left(\rho-x_{2}, \sigma y_{2}\right)$. However, making use of the techniques of Section 3 , we do not need to perform the final powering, as we presume that the output will be stored and transmitted in compressed form (Algorithm 2).

\subsection{Cost analysis}

Let $M$ denote the cost of an $\mathbb{F}_{q}$ multiplication. Each iteration of the loop requires $2 M$ to compute $\mu^{2}$ and $y_{1} y_{2}$, and an $\mathbb{F}_{q^{6}}$ multiplication to compute $f \cdot g$. Since a generic $\mathbb{F}_{q^{6}}$ multiplication costs $18 M$, Scott and Barreto [46] reckon that besides the necessary cubings and cube roots, each loop iteration costs $20 M$. However, in each iteration $g$ is sparse (that is, not all of its terms are non-trivial), one can exploit this to reduce the cost of multiplying $g$ and $f$, which is not sparse in general, to $13 M$. This total of $15 M$ improves on the tracebased method suggested by Scott and Barreto. In fact, one can reduce the cost for each loop 


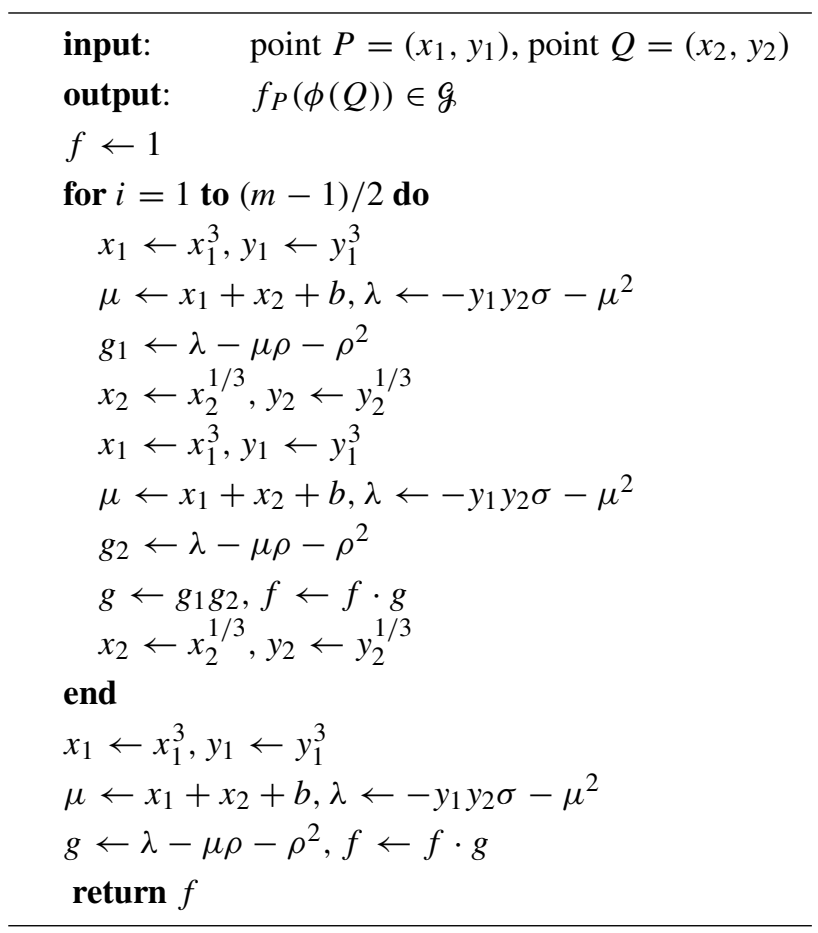

Algorithm 3: A refined Duursma-Lee algorithm

iteration in the ordinary Duursma-Lee algorithm to just $14 M$, by unrolling the main loop and better exploiting the sparsity of $g$.

We demonstrate this technique in Algorithm 3, which provides a saving since in each loop, multiplying $g_{1}$ by $g_{2}$ costs only $6 M$. Multiplying $g$ by $f$ in each loop costs $18 M$ since they are both generic $\mathbb{F}_{q^{6}}$ elements. Both $\mu^{2}$ and $y_{1} y_{2}$ are computed twice in each loop: once for $g_{1}$ and once for $g_{2}$. In total, the cost is therefore $(6 M+4 M)(m-1) / 2+18 M(m-3) / 2+13 M=$ $14 m M-19 M$, which is equivalent to about $14 M$ per loop iteration of Algorithm 2.

This cost analysis ignores the cost of computing cubings and cube roots. Because of the large number of times each of these operations is invoked, it has been suggested that one should use normal bases to accommodate them efficiently, since they are then implemented using cyclic shifts. Normal bases are well-studied in even characteristic, but for characteristic three one cannot construct optimal, type-one normal bases with prime extension degree $[19,38]$, although type-two bases are available for some values of $m$. As a result, the cost of general multiplication in software is relatively large, even when variations of high-performance methods in characteristic two are used [40, 37]. For example, we found that when $m=239$, normal basis multiplication is between two and three times slower than a polynomial basis multiplication. However, in hardware implementations on a smart-card for example, normal bases still seem the obvious choice since they can match the multiplication speed of polynomial bases while offering inexpensive cube and cube-root operations, although perhaps at the cost of flexibility.

To reduce the cost of computing cube roots using a polynomial basis, we observe that the successive cube roots of $x_{2}$ and $y_{2}$ can be computed more easily in reverse order and stored for the duration of the algorithm. Since for any $x_{2} \in \mathbb{F}_{q}$, we have $x_{2}=x_{2}^{3^{m}}$, the 
required values $x_{2}^{1 / 3^{i}}$ can be computed as $x_{2}^{3^{m-i}}$, and thus one does not need to compute any cube roots at all. The memory requirement for this is only about $2^{-11} \mathrm{~m}^{2} \mathrm{~Kb}$, and the time taken is just the cost of $2 m$ cubings. If memory is at a premium, one can reduce this to about $2^{-4.5} \mathrm{~m}^{3 / 2} \mathrm{~Kb}$ with double the number of cubings, using further loop-unrolling and pebbling strategies.

REMARK 3. As already mentioned, Scott and Barreto's method [46] is effectively a change of basis and not a compressed method of computing a pairing. Hence it is unsurprising that the loop-unrolling strategy of Algorithm 3 can be used to reduce the cost of the trace method given there, as was kindly pointed out by Barreto in a personal communication.

REMARK 4. Scott and Barreto [46] suggested an open problem, asking whether it is possible to perform the pairing computation directly in compressed form for some compression factor of at least 3 on ordinary (non-supersingular) curves in characteristic $p>3$. A compression factor larger than 3 is extremely unlikely. For pairing-based applications, the desirable extension degrees in the near future are likely to remain small, and no larger than twenty. By Lemma 1, the maximum compression factor possible for a given extension degree $n$ is $n / \phi(n)$, and for $n<20$, this maximum is three, which has already been achieved for the modified Tate pairing.

Table 3: Pairing and exponentiation timings

\begin{tabular}{lcccccc}
\hline \multicolumn{1}{c}{} & $\mathbb{F}_{3^{79}}$ & $\mathbb{F}_{397}$ & $\mathbb{F}_{3163}$ & $\mathbb{F}_{3193}$ & $\mathbb{F}_{3239}$ & $\mathbb{F}_{3353}$ \\
\hline Pairing & & & & & & \\
BKLS & $13.96 \mathrm{~ms}$ & $23.60 \mathrm{~ms}$ & $79.11 \mathrm{~ms}$ & $123.21 \mathrm{~ms}$ & $179.30 \mathrm{~ms}$ & $527.56 \mathrm{~ms}$ \\
Algorithm 3 & $4.67 \mathrm{~ms}$ & $8.41 \mathrm{~ms}$ & $29.26 \mathrm{~ms}$ & $45.67 \mathrm{~ms}$ & $65.73 \mathrm{~ms}$ & $197.58 \mathrm{~ms}$ \\
Exponentiation in $G_{l}$ & & & & & \\
Method 1 & $3.65 \mathrm{~ms}$ & $6.14 \mathrm{~ms}$ & $20.98 \mathrm{~ms}$ & $33.21 \mathrm{~ms}$ & $44.72 \mathrm{~ms}$ & $130.27 \mathrm{~ms}$ \\
Method 2 & $4.57 \mathrm{~ms}$ & $7.25 \mathrm{~ms}$ & $21.53 \mathrm{~ms}$ & $31.61 \mathrm{~ms}$ & $43.56 \mathrm{~ms}$ & $119.16 \mathrm{~ms}$ \\
Method 3 & $3.67 \mathrm{~ms}$ & $5.79 \mathrm{~ms}$ & $17.85 \mathrm{~ms}$ & $26.69 \mathrm{~ms}$ & $36.45 \mathrm{~ms}$ & $101.75 \mathrm{~ms}$ \\
Method 4 & $3.06 \mathrm{~ms}$ & $5.10 \mathrm{~ms}$ & $16.55 \mathrm{~ms}$ & $24.67 \mathrm{~ms}$ & $34.74 \mathrm{~ms}$ & $99.56 \mathrm{~ms}$
\end{tabular}

Exponentiation in $g$

\begin{tabular}{lcccccc} 
Method 1 & $2.55 \mathrm{~ms}$ & $4.27 \mathrm{~ms}$ & $14.15 \mathrm{~ms}$ & $21.67 \mathrm{~ms}$ & $30.69 \mathrm{~ms}$ & $88.06 \mathrm{~ms}$ \\
Method 2 & $2.62 \mathrm{~ms}$ & $5.21 \mathrm{~ms}$ & $13.21 \mathrm{~ms}$ & $20.38 \mathrm{~ms}$ & $26.97 \mathrm{~ms}$ & $74.90 \mathrm{~ms}$ \\
Method 3 & $3.69 \mathrm{~ms}$ & $4.72 \mathrm{~ms}$ & $15.78 \mathrm{~ms}$ & $22.96 \mathrm{~ms}$ & $37.96 \mathrm{~ms}$ & $73.29 \mathrm{~ms}$ \\
Method 4 & $2.32 \mathrm{~ms}$ & $4.07 \mathrm{~ms}$ & $11.84 \mathrm{~ms}$ & $17.63 \mathrm{~ms}$ & $24.73 \mathrm{~ms}$ & $69.30 \mathrm{~ms}$ \\
\multicolumn{1}{l}{ Point Multiplication in E $\left(\mathbb{F}_{q}\right)$} & & & & \\
Method 1 & $1.83 \mathrm{~ms}$ & $3.11 \mathrm{~ms}$ & $10.62 \mathrm{~ms}$ & $16.94 \mathrm{~ms}$ & $24.11 \mathrm{~ms}$ & $69.78 \mathrm{~ms}$ \\
Method 2 & $1.72 \mathrm{~ms}$ & $2.84 \mathrm{~ms}$ & $9.47 \mathrm{~ms}$ & $14.73 \mathrm{~ms}$ & $21.15 \mathrm{~ms}$ & $60.70 \mathrm{~ms}$ \\
Method 3 & $1.82 \mathrm{~ms}$ & $3.01 \mathrm{~ms}$ & $9.66 \mathrm{~ms}$ & $14.95 \mathrm{~ms}$ & $21.19 \mathrm{~ms}$ & $58.70 \mathrm{~ms}$ \\
Method 4 & $1.18 \mathrm{~ms}$ & $1.95 \mathrm{~ms}$ & $8.11 \mathrm{~ms}$ & $12.75 \mathrm{~ms}$ & $19.04 \mathrm{~ms}$ & $55.93 \mathrm{~ms}$ \\
\hline
\end{tabular}




\section{Implementation results}

In order to provide some concrete idea of the practical cost of our own and other methods, we implemented the proposed field arithmetic, pairing algorithms and exponentiation methods. We used a GCC 3.3 compiler suite to build our implementation, and ran timing experiments on a Linux-based PC incorporating a $2.80 \mathrm{GHz}$ Intel Pentium 4 processor. The entire system was constructed in $\mathrm{C}++$. We accept that further performance improvements could be made through aggressive profiling and optimisation, but we are confident that our results are representative of the underlying algorithms and allow a comparison between them.

Table 3 shows the result of timing this implementation using a variety of different base field sizes. In the pairing section, 'Algorithm 3' refers to the augmented version of DuursmaLee presented in this paper, with the cube-root precomputation strategy and the loop unrolling. The BKLS method is included as a reference. We do not include timings for the methods of [46] since our operation count clearly shows they will be slower than our alternatives. Table 4 gives timings for the underlying field operations.

Table 4: Timings for field operations

\begin{tabular}{|c|c|c|c|c|c|c|}
\hline & $\mathbb{F}_{3^{79}}$ & $\mathbb{F}_{3^{97}}$ & $\mathbb{F}_{3163}$ & $\mathbb{F}_{3193}$ & $\mathbb{F}_{3239}$ & $\mathbb{F}_{3353}$ \\
\hline \multicolumn{7}{|l|}{$\mathbb{F}_{q}$} \\
\hline Add & $0.55 \mu s$ & $0.53 \mu s$ & $0.58 \mu s$ & $0.63 \mu s$ & $0.61 \mu s$ & $0.64 \mu s$ \\
\hline Square & $4.42 \mu s$ & $6.07 \mu s$ & $12.99 \mu s$ & $16.48 \mu s$ & $19.48 \mu s$ & $40.97 \mu s$ \\
\hline Cube & $0.85 \mu s$ & $0.84 \mu s$ & $0.96 \mu s$ & $1.26 \mu s$ & $1.24 \mu s$ & $1.77 \mu s$ \\
\hline Invert & $23.18 \mu s$ & $33.26 \mu \mathrm{s}$ & $70.10 \mu s$ & $97.20 \mu s$ & $136.86 \mu s$ & $303.27 \mu s$ \\
\hline Multiply & $4.06 \mu s$ & $6.02 \mu s$ & $12.80 \mu s$ & $17.83 \mu s$ & $19.42 \mu s$ & $43.11 \mu s$ \\
\hline \multicolumn{7}{|l|}{$\mathbb{F}_{q^{3}}$} \\
\hline Add & $0.60 \mu s$ & $0.60 \mu s$ & $0.80 \mu s$ & $0.90 \mu s$ & $0.90 \mu s$ & $0.50 \mu s$ \\
\hline Cube & $2.10 \mu s$ & $2.10 \mu s$ & $2.30 \mu s$ & $2.50 \mu s$ & $3.20 \mu s$ & $4.20 \mu s$ \\
\hline Invert & $65.00 \mu s$ & $94.70 \mu s$ & $204.40 \mu s$ & $275.90 \mu s$ & $350.60 \mu s$ & $741.80 \mu s$ \\
\hline Frobenius & $1.10 \mu s$ & $0.90 \mu s$ & $1.10 \mu s$ & $1.00 \mu s$ & $1.30 \mu s$ & $1.40 \mu s$ \\
\hline Multiply & $26.10 \mu s$ & $37.80 \mu s$ & $74.20 \mu s$ & $98.00 \mu s$ & $115.50 \mu s$ & $249.00 \mu s$ \\
\hline \multicolumn{7}{|l|}{$\mathbb{F}_{q^{6}}$} \\
\hline Add & $0.90 \mu s$ & $0.90 \mu s$ & $0.90 \mu s$ & $1.10 \mu s$ & $1.00 \mu s$ & $1.10 \mu s$ \\
\hline Cube & $2.80 \mu s$ & $4.60 \mu s$ & $4.40 \mu s$ & $4.00 \mu s$ & $5.00 \mu s$ & $5.60 \mu s$ \\
\hline Invert & $165.50 \mu s$ & $237.20 \mu s$ & $497.40 \mu s$ & $670.10 \mu s$ & $817.10 \mu s$ & $1709.50 \mu s$ \\
\hline Frobenius & $2.00 \mu s$ & $2.10 \mu s$ & $1.90 \mu s$ & $2.00 \mu s$ & $2.10 \mu s$ & $2.10 \mu s$ \\
\hline Multiply & $75.70 \mu s$ & $106.10 \mu s$ & $227.10 \mu s$ & $296.80 \mu s$ & $347.30 \mu s$ & $745.10 \mu s$ \\
\hline
\end{tabular}


We note first that our implementation of Algorithm 3 is between two to three times faster than the BKLS algorithm. With regard to exponentiation, Method 4 is the most efficient for all field sizes and in all three groups, and in $g$ is nearly twice as fast as Method 1 in $G_{l}$. An early estimate of Koblitz [30] states that the ratio of the time required for an exponentation in $\mathbb{F}_{q^{6}}$ to the time required for a point multiplication in $E\left(\mathbb{F}_{q}\right)$ is 12 ; our results demonstrate that for fields of a cryptographic size, this value is in fact closer to 1.3. Thus the techniques from [52], together with the fast multiplication in $g$, considerably improve the efficiency of post-pairing arithmetic.

We conceed that while we have not implemented Koblitz's complex multiplication exponentiation method, due to the estimated large preprocessing time required, we do not think that it would significantly affect this comparison.

Furthermore, due to our direct inversion method, the ratio of inversion time to multiplication time in $\mathbb{F}_{q^{3}}$ is under three for all field sizes. This means that our compression method in $g$ costs roughly $4 / 3$ multiplications in $\mathbb{F}_{q^{6}}$, and is therefore also very efficient.

\section{Conclusion and open problems}

We have shown how to take advantage of the quotient group to which a pairing value naturally belongs in order to speed up exponentiations, and to obtain fast compression of pairing values. We have also proposed some simple refinements to the Duursma-Lee algorithm to improve efficiency. Our results strongly indicate that there are definite advantages to implementing pairing-based cryptographic protocols in characteristic three: the oftenquoted value of ten for the ratio of the speed of a pairing evaluation to a point multiplication on the curve is really closer to three or four.

Some issues remain. One could certainly improve the exponentiation times for all three groups if there existed an efficiently computable ternary analogue of the Joint Sparse Form [50]. With regard to side channel attacks, such a method may be undesirable since one cannot render cubing and multiplication in characteristic-three fields indistinguishable without a serious detriment to performance. As such, a cube-and-multiply-always method using the exponent splitting of Method 4 will halve the cost of a secure full-length expansion.

Also, the exact security of the discrete logarithm problem in characteristic three using the ternary analogue of Coppersmith's method has yet to be investigated $[\mathbf{1 0 , 1 1 ]}$. Preliminary research into this problem using Adleman's Function Field Sieve has been conducted [23, 24], but the problem should still be considered open.

Lastly, do there exist methods for faster pairing evaluation using MNT curves, and how might they compare to those presented here?

Acknowledgements The authors would like to thank the anonymous referee, Paulo Barreto, Steven Galbraith, Keith Harrison, Karl Rubin, Mike Scott, Alice Silverberg, Nigel Smart and Fré Vercauteren for many helpful comments and fruitful discussions.

\section{References}

1. P. Barreto, S. Galbraith, C. Ó hÉigeartaigh and M. Scott, 'Efficient pairing computation on supersingular abelian varieties', Cryptology ePrint Archive, Report 2004/375, http://eprint.iacr.org/2004/375.75 
2. P. Barreto, H. Kim, B. Lynn and M. Scott, 'Efficient algorithms for pairing-based cryptosystems', Advances in cryptology (CRYPTO 2002), Lecture Notes in Comput. Sci. 2442 (Springer, 2002) 354-368. 64, 65, 66

3. D. BONEH and X. BOYEN, 'Efficient selective-ID secure identity-based encryption without random oracles', Advances in cryptology (EUROCRYPT 2004), Lecture Notes in Comput. Sci. 3027 (Springer, 2004) 223-238. 65

4. D. Boneh, X. Boyen and H. Shacham, 'Short group signatures', Advances in cryptology (CRYPTO 2004), Lecture Notes in Comput. Sci. 3152 (Springer, 2004) 41-55. 65

5. D. Boneh and M. Franklin, 'Identity-based encryption from the Weil pairing', SIAM J. Comput., 32 (2003) 586-615. 64, 65

6. D. Boneh, B. Lynn and H. Shacham, 'Short signatures from the Weil pairing', Advances in cryptology (ASIACRYPT 2001), Lecture Notes in Comput. Sci. 2248 (Springer, 2001) 514-532. 64, 72

7. W. Bosma, J. Hutton and E. Verheul, 'Looking beyond XTR', Advances in cryptology (ASIACRYPT 2002), Lecture Notes in Comput. Sci. 2501 (Springer, 2002) 46-63. 67

8. W. CLARK and J. LiAng, 'On arithmetic weight for a general radix representation of integers', IEEE Trans. Inform. Theory 19 (1973) 823-826. 72

9. H. Cohen, A. Miyaji and T. Ono, 'Efficient elliptic curve exponentiation using mixed coordinates', Advances in cryptology (ASIACRYPT 1998), Lecture Notes in Comput. Sci. 1514 (Springer, 1998) 51-65. 69

10. D. Coppersmith, 'Evaluating logarithms in $\mathrm{GF}\left(2^{n}\right)$ ', 16th ACM Symp. Theory of Computing (1984) 201-107. 81

11. D. Coppersmith, 'Fast evaluation of logarithms in fields of characteristic two', IEEE Trans. Inform. Theory, 30 (July 1984) 587-594. 81

12. R. Dutta, R. BARUa and P. SARKar, 'Pairing-based cryptographic protocols: a survey', Cryptology ePrint Archive, Report 2004/064, http://eprint.iacr.org/2004/064.64

13. I. DuURsma and H. LEE, 'Tate pairing implementation for hyperelliptic curves $y^{2}=$ $x^{p}-x+d$, Advances in cryptology (ASIACRYPT 2003), Lecture Notes in Comput. Sci. 2894 (Springer, 2003) 111-123. 64, 66

14. T. ElGamal, 'A public key cryptosystem and a signature scheme based on discrete logarithms', IEEE Trans. Inform. Theory 31 (1985) 469-472. 67

15. G. FreY and H. RuCK, 'A remark concerning $m$-divisibility and the discrete logarithm problem in the divisor class group of curves', Math. Comput. 62 (1994) 865-874. 66

16. S. Galbraith, Supersingular curves in cryptography',Advances in cryptology (ASIACRYPT 2001), Lecture Notes in Comput. Sci. 2248 (Springer, 2001) 495-513. 65

17. S. Galbraith, K. Harrison and D. Soldera, 'Implementing the Tate pairing', Proc. ANTS V, Lecture Notes in Comput. Sci. 2369 (2002) 324-337. 64, 65, 66, 75

18. R. Gallant, J. Lambert and S. Vanstone, 'Faster point multiplication on elliptic curves with efficient endomorphisms', Advances in cryptology (CRYPTO 2001), Lecture Notes in Comput. Sci. 2139 (Springer, 2001) 190-200. 73 
19. S. GAO, 'Normal bases over finite fields', $\mathrm{PhD}$ Thesis, Waterloo University, 1993. 78

20. P. GAUDRY, 'Index calculus for abelian varieties and the elliptic curve discrete logarithm problem', Cryptology ePrint Archive, Report 2004/073,

http: //eprint.iacr.org/2004/073. 72

21. C. Gentry, 'Certificate-based encryption and the certificate revocation problem', Advances in cryptology (EUROCRYPT 2003), Lecture Notes in Comput. Sci. 2656 (Springer, 2003) 272-293. 65

22. P. Golle and A. Juels, 'Dining cryptographers revisited', Advances in cryptology (EUROCRYPT 2004), Lecture Notes in Comput. Sci. 3027 (Springer, 2004) 456-473. 65

23. R. Granger, 'Estimates for discrete logarithm computations in finite fields of small characteristic', Cryptography and coding, Lecture Notes in Comput. Sci. 2898 (Springer, 2003) 190-206. 81

24. R. Granger, A. Holt, D. Page, N. Smart and F. Vercauteren, 'Function field sieve in characteristic three', Proc. ANTS VI, Lecture Notes in Comput. Sci. 3076 (Springer, 2004) 223-234. 81

25. R. Granger, D. Page and M. Stam, 'A Comparison of CEILIDH and XTR', Proc. ANTS VI, Lecture Notes in Comput. Sci. 3076 (Springer, 2004) 235-249. 65, 70, 71, 75

26. R. Granger, D. Page and M. Stam, 'On small characteristic algebraic tori in pairingbased cryptography, Cryptology ePrint Archive, Report 2004/132, http: / /eprint.iacr.org/2004/132.75

27. K. Harrison, D. Page and N. P. Smart, 'Software implementation of finite fields of characteristic three, for use in pairing based cryptosystems', LMS J. Comput. Math., 5 (2002) 181-193, http: / / www. lms . ac . uk/ jcm/5/lms 2002-002. 75

28. F. Hess, Efficient identity based signature schemes based on pairings', Selected areas in cryptography (SAC 2002), Lecture Notes in Comput. Sci. 2595 (Springer, 2003) 310-324. 65

29. A. Joux, 'A one round protocol for tripartite Diffie-Hellman', Proc. ANTS IV, Lecture Notes in Comput. Sci. 1838 (Springer, 2000) 385-394. 64, 72

30. N. Koblitz, 'An elliptic curve implementation of the finite field digital signature algorithm', Advances in cryptology (CRYPTO 98), Lecture Notes in Comput. Sci. 1462 (Springer, 1998) 327-337. 73, 81

31. S. Kwon, 'Efficient Tate pairing computation for elliptic curves over binary fields', Proc. ACISP 2005, Lecture Notes in Comput. Sci. 3574 (Springer, 2005) 134-145. 75

32. A. Lenstra, 'Using cyclotomic polynomials to construct efficient discrete logarithm cryptosystems over finite fields', Proc. ACISP97, Lecture Notes in Comput. Sci. 1270 (Springer, 1997) 127-138. 67

33. A. Lenstra and E. Verheul, 'The XTR public key system', Advances in cryptology (CRYPTO 2000), Lecture Notes in Comput. Sci. 1880 (Springer, 2000) 1-19. 64, 67, 75

34. J. López and R. DAHAB, 'High speed software multiplication in $\mathbb{F}_{2^{m}}$ ', Progress in cryptography (INDOCRYPT 2000), Lecture Notes in Comput. Sci. 1977 (Springer, 2000) 203-212. 76 
35. A. J. Menezes, P. C. Van Oorschot and S. A. Vanstone, Handbook of applied cryptography (CRC Press, 1997). 73

36. V. Miller, 'Short programs for functions on curves', unpublished manuscript, 1986, available from http://crypto.stanford.edu/miller/miller.pdf. 65

37. P. NING and Y. L. YIN, 'Efficient software implementation for finite field multiplication in normal basis', Information and communications security (ICICS), Lecture Notes in Comput. Sci. 2229 (Springer, 2001) 177-188. 78

38. M. NöcKER, 'Data structures for parallel exponentiation in finite fields', PhD Thesis, Universität Paderborn, 2001. 78

39. G. Pohlig and M. Hellman, 'An improved algorithm for computing discrete logarithms over $\mathrm{GF}(p)$ and its cryptographic significance', IEEE Trans. Inform. Theory 24 (1978) 106-110. 67

40. A. Reyhani-Masoleh and M. A. Hasan, 'Fast normal basis multiplication using general purpose processors', Selected areas in cryptography (SAC 2001), Lecture Notes in Comput. Sci. 2259 (Springer, 2001) 230-244. 78

41. K. Rubin and A. Silverberg, 'Supersingular abelian varieties in cryptology', Advances in cryptology (CRYPTO 2002), Lecture Notes in Comput. Sci. 2442 (Springer, 2002) 336-353. 72

42. K. Rubin and A. Silverberg, 'Torus-based cryptography', Advances in cryptology (CRYPTO 2003), Lecture Notes in Comput. Sci. 2729 (Springer, 2003) 349-365. 64, $65,67,68,70,71$

43. K. Rubin and A. Silverberg, 'Using primitive subgroups to do more with fewer bits', Algorithm number theory (ANTS-VI), Lecture Notes in Comput. Sci. 3076 (Springer, 2004) 18-41. 72

44. R. Sakai, K. Ohgishi and M. Kasahara, 'Cryptosystems based on pairings', Symposium on Cryptography and Information Security 2000 (SCIS2000), Okinawa, Japan, Jan 26-28, 2000. 64

45. M. Sсотт, 'Authenticated ID-based key exchange and remote log-in with insecure token and PIN number', Cryptology ePrint Archive, Report 2002/164,

http: / /eprint.iacr.org/2002/164.65

46. M. ScotT and P. BARreto, 'Compressed pairings', Advances in cryptology (CRYPTO 2004), Lecture Notes in Comput. Sci. 3152 (Springer, 2004) 140-156. 64, 66, 74, 77, 79,80

47. I. Semaev, 'Summation polynomials and the discrete logarithm problem on elliptic curves', Cryptology ePrint Archive, Report 2004/031,

http://eprint.iacr.org/2004/031. 72

48. J. Silverman, The arithmetic of elliptic curves, Grad. Texts in Math. 106 (Springer, 1986). 66

49. P. Smith and C. Skinner, 'A public-key cryptosystem and a digital signature system based on the Lucas function analogue to discrete logarithms', Advances in cryptology (ASIACRYPT 1995), Lecture Notes in Comput. Sci. 917 (Springer, New York, 1995) 357-364. 64, 75

50. J. A. Solinas, 'Low-weight binary representations for pairs of integers', University of Waterloo, Technical Report CORR 2001-41. 81 
51. M. StAm and A. Lenstra, 'Speeding up XTR', Advances in cryptology (ASIACRYPT 2001), Lecture Notes in Comput. Sci. 2248 (Springer, 2001) 125-143. 75

52. M. StAm and A. Lenstra, 'Efficient subgroup exponentiation in quadratic and sixth degree extensions', Cryptographic hardware and embedded systems (CHES 2002), Lecture Notes in Comput. Sci. 2523 (Springer, 2002) 318-332. 72, 73, 81

53. E. G. Straus, 'Problems and solutions: (5125) Addition chains of vectors', Amer. Math. Monthly 71 (1964) 806-808. 73

54. V. E. VosKRESENSKIĬ, Algebraic groups and their birational invariants, Transl. Math. Monogr. 179 (Amer. Math. Soc., Providence, RI, 1998). 67

R. Granger grangerecs.bris.ac.uk

D. Page pagedcs.bris.ac.uk

http://www.cs.bris.ac.uk/ page/

University of Bristol

Department of Computer Science

Merchant Venturers Building

Woodland Road

Bristol, BS8 1UB

United Kingdom

M. Stam martijn.stam@epfl.ch

EPFL - IC -LACAL

Station 14, INJ 3.33

CH-1015 Lausanne

Switzerland 